Research Paper

\title{
Upregulation of Enzymes involved in ISGylation and Ubiquitination in patients with hepatocellular carcinoma
} \author{
Velavan ${ }^{4,5,6}$, Nguyen Linh Toan² \\ 1. Institute of Biomedicine and Pharmacy, Vietnam Military Medical University, Hanoi, Vietnam \\ 2. Department of Pathophysiology, Vietnam Military Medical University, Hanoi, Vietnam \\ 3. 108 Military Central Hospital, Hanoi, Vietnam \\ 4. Vietnamese-German Center of Excellence in Medical Research, Hanoi, Vietnam \\ 5. Institute of Tropical Medicine, University of Tübingen, Tübingen, Germany \\ 6. Duy Tan University, Da Nang, Vietnam \\ 7. Hai Duong Medical Technical University, Hai Duong, Vietnam
}

Hoang Van Tong1,2, ${ }^{\circledR}$, Nghiem Xuan Hoan 3,4, Mai Thanh Binh $3,4,5$, Dao Thanh Quyen 3,4, Christian G. Meyer ${ }^{4,5,6}$, Dinh Thi Thu Hang1, Dinh Thi Dieu Hang7, Ho Anh Son ${ }^{1,2}$, Hoang Van Luong1, Nghiem Duc Thuan¹, Nguyen Truong Giang1, Do Quyet1, Mai Hong Bang3, Le Huu Song3,4, Thirumalaisamy P.

$\triangle$ Corresponding author: Dr. Hoang Van Tong, PhD, Institute of Biomedicine and Pharmacy, Vietnam Military Medical University, 222 Phung Hung, Ha Dong, Hanoi, Vietnam. Tel.: +84 868358089; E-mail: hoangvantong@vmmu.edu.vn

(C) The author(s). This is an open access article distributed under the terms of the Creative Commons Attribution License (https://creativecommons.org/licenses/by/4.0/). See http://ivyspring.com/terms for full terms and conditions.

Received: 2019.08.31; Accepted: 2019.12.12; Published: 2020.01.20

\begin{abstract}
Background: ISGylation is the conjugation of ISG15 with target proteins. ISGylation occurs through an enzymatic cascade, which is similar to that of ubiquitination. Through ISGylation, ISG 15 can bind to proteins involved in cell proliferation and differentiation, thus promoting genesis and progression of malignancies. The present study aims to investigate expression of genes involved in ISGylation and ubiquitination in patients with hepatocellular carcinoma and to correlate gene expression with clinical laboratory parameters of these patients.
\end{abstract}

Methods: mRNA expression of genes encoding enzymes involved in the ISGylation process (EFP, HERC5, UBAI, UBC and USPI8) was evaluated by quantitative real-time PCR in 38 pairs of tumour and adjacent non-tumour tissues from patients with hepatocellular carcinoma and correlated with distinct clinical laboratory parameters.

Results: Relative mRNA expression of EFP, HERC5, UBAI and USPI8 was significantly higher in tumour tissues compared to adjacent non-tumour tissues $(P=0.006 ; 0.012 ; 0.02$ and 0.039 , respectively). The correlation pattern of mRNA expression between genes in the tumours differed from the pattern in adjacent non-tumour tissues. Relative expression of EFP, HERC5 and UBAI in adjacent non-tumour tissues was positively associated with direct bilirubin levels (Spearman's rho $=0.31,0.33$ and $0.45 ; P=0.06,0.05$ and 0.01 , respectively) and relative expression of USPI 8 in adjacent non-tumour tissues correlated negatively with ALT levels (Spearman's rho $=-0.33, P=0.03$ ).

Conclusions: EFP, HERC5, UBAI, and USPI8 genes are upregulated in tumour tissues of patients with $\mathrm{HCC}$ and, thus, may be associated with the pathogenesis of hepatocellular carcinoma.

Key words: hepatocellular carcinoma, Interferon-stimulated gene 15 (ISG15), ISGylation, E3 ligase, ubiquitin-specific protease 18 (USP18)

\section{Introduction}

Hepatitis B virus (HBV) infection is responsible for up to $90 \%$ of hepatocellular carcinoma (HCC) cases, especially in regions with a high prevalence of HBV infection including Vietnam [1, 2]. HCC accounts for $75-85 \%$ of primary liver cancer and is the fourth leading cause of cancer-related deaths. Annually, 841,000 new cases and 782,000 deaths are reported worldwide [2]. The number of HCC-related 
deaths is increasing and estimated to reach a prevalence of one million by 2030 [3]. HBV infection can cause HCC through direct interaction of viral factors (e.g. HBV-DNA, HBx protein) with host cells, causing instability of human chromosomes and induction of oncogenes. Indirect mechanisms result from inflammation of hepatocytes, fibrosis and modification of cellular signalling involving the JAK/STAT and Wnt/ $\beta$-catenin pathways [4].

In response to $\mathrm{HBV}$ infection, immune cells secrete interferon (IFN) and other inflammatory cytokines. Subsequently, cellular signalling pathways are activated and IFN-stimulated genes (ISGs) are induced [5]. Among the ISGs, ISG15 is a key effector molecule of the innate immune system. ISG15 is induced by type I interferon and lipopolysaccharides and has antiviral activity against many viruses [6]. It plays also an important role in several immunomodulatory activities, including induction of NK cell proliferation, triggering $\mathrm{T}$ cell responses, augmentation of lymphokine-activated killer (LAK) activity, stimulation of IFN- $\gamma$ production, inducing dendritic cell (DC) maturation and acting as a chemotactic factor for neutrophils [7, 8]. ISG15 exists both as a free form and/or in conjugation with other proteins. The conjugation of ISG15, ISGylation, occurs through an enzymatic cascade through which ISG15 can modulate the functions of pathogenic and host proteins and affect downstream signalling pathways [9]. Initially, the activation of the E1 enzyme (ubiquitin-like modifier-activating enzyme 7; UBA1 or UbE1L) forms a thioester bond with free ISG15 in an ATP-dependent manner [7]. ISG15 is then transferred to the ubiquitin/ISG15-conjugating enzyme E2 L6 (UBE2L6 or UbcH8). Finally, E3 ligases catalyze the conjugation of ISG15 with lysine residues of target substrate proteins, a process which may be reversible (deISGylation) by the activity of the ubiquitin-specific protease 18 (USP18) [6].

ISGylation has multiple functions, including inhibition of release of exosomes, downregulation of protein translation and regulation of distinct protein functions [10]. In cancer, ISGylation has been shown to target p53, a tumour protein, and control its stability and functionality [11]. Through ISGylation, ISG15 is associated with the formation, development and progression of several malignancies such as breast, lung and ovarian cancers [12-14]. Several enzymes involved in the ISGylation process are associated with cancers in both an ISGylation-dependent and ISGylation-independent manner. Particularly, the HECT domain and the RCC1-like domain-containing protein 5 (HERC5) and estrogen-responsive finger protein (EFP or TRIM25), known as E3 ligase of ISGylation have been shown to be associated with liver, breast prostate, endometrial and ovarian cancers [15-18]. USP18 is also involved in the development of breast, lung and liver cancers [19-21]. A study has indicated that USP18 contributes to controlling carcinogenesis, as loss of USP18 function increases apoptosis and decreases cell proliferation by destabilization of the cyclin D1 protein [22].

It remains, however, unclear so far whether the EFP, HERC5 and USP18 genes contribute to tumourigenesis through ISG15/ISGylation or other mechanisms. In addition, the exact role of ISG15 and other key enzymes involved in ISGylation in the formation and development of HBV-related HCC is still unclear. In the present study, we aim to investigate the expression profiles of genes encoding enzymes involved in the ISGylation process (EFP, HERC5, UBA1, UBC USP18) in patients with HCC and their association with clinical outcomes.

\section{Methods}

\section{Study subjects}

The study included 38 patients with HCC who underwent surgery at the 108 Military Central Hospital in Hanoi, Vietnam. The patients were diagnosed according to the guidelines of the American Association for the Study of Liver Diseases (AASLD) for management of HCC [21]. HCC was confirmed by computed tomography (CT) imaging and/or by histology and classified based on the Barcelona Clinic Liver Cancer (BCLC) classification system [26]. HBV and HCV infections were assessed in order to determine the etiology of the disease. Liver tissue specimens collected from the HCC patients included tumour and adjacent non-tumour specimens; the samples were and frozen at $-80^{\circ} \mathrm{C}$ until further use. Patients were further grouped as Child-A, Child-B or Child-C according to Child-Pugh scores [22]. Laboratory parameters involving blood counts, total and direct bilirubin, prothrombin, albumin, alanine transaminase (ALT), aspartate transaminase (AST), and the tumour marker alpha-fetoprotein (AFP) were assessed by routine laboratory tests. The baseline characteristics of the 38 HCC patients including age, gender and the laboratory results are shown in Table 1.

All details of the study were explained to the participants and informed written consent was obtained before taking samples. All clinical procedures and experiments were performed in accordance with applying guidelines and regulations. The study was approved by the Institutional Review Board of the 108 Central Hospital, Hanoi, Vietnam. 
Table 1: Characteristics of $38 \mathrm{HCC}$ patients

\begin{tabular}{|c|c|}
\hline Characteristics & n (\%) \\
\hline \multicolumn{2}{|l|}{ Age (years) } \\
\hline$<40$ & $4 / 38(10.5)$ \\
\hline $40-60$ & $25 / 38(65.8)$ \\
\hline$>60$ & $9 / 38(23.7)$ \\
\hline \multicolumn{2}{|l|}{ Gender } \\
\hline Male & $34 / 38(89.5)$ \\
\hline Female & $4 / 38(10.5)$ \\
\hline \multicolumn{2}{|l|}{ Etiology } \\
\hline $\mathrm{HBV}$ & $29 / 38(76.3)$ \\
\hline $\mathrm{HCV}$ & $1 / 38(2.6)$ \\
\hline Non-HBV/HCV & $8 / 38(21.1)$ \\
\hline \multicolumn{2}{|l|}{ BCLC staging Classification } \\
\hline Stage A & $31 / 38(81.6)$ \\
\hline Stage B & $7 / 38(18.4)$ \\
\hline Stage C and D & $0 / 38(0)$ \\
\hline \multicolumn{2}{|l|}{ Child Pugh Classification } \\
\hline Child A & $18 / 38(47.4)$ \\
\hline Child B & $6 / 38(15.8)$ \\
\hline Unclassified & $14 / 38(36.8)$ \\
\hline Clinical parameters & Median [Range] \\
\hline WBC $\left(x 10^{6} / \mathrm{ml}\right)$ & $7.3[3.88-15.4]$ \\
\hline $\mathrm{RBC}\left(\times 10^{3} / \mathrm{ml}\right)$ & $4.46[2.7-5.8]$ \\
\hline HCT (\%) & 131 [109-151] \\
\hline $\operatorname{PLT}\left(10^{3} / \mathrm{ml}\right)$ & $170.5[97-497]$ \\
\hline HBV-DNA & NA \\
\hline AST (IU/ml) & $54.5[16-250]$ \\
\hline $\operatorname{ALT}(\mathrm{IU} / \mathrm{ml})$ & $76[21-476]$ \\
\hline Total Bilirubin $(\mu \mathrm{mol} / \mathrm{l})$ & $18.8[8.2-50]$ \\
\hline Direct Bilirubin $(\mu \mathrm{mol} / \mathrm{l})$ & $5.7[1-39.4]$ \\
\hline Prothrombin (\% of standard) & $86[50-125]$ \\
\hline Protein $(\mathrm{g} / \mathrm{l})$ & $65[52-74]$ \\
\hline Allbumin (g/l) & $35[24-45]$ \\
\hline $\operatorname{AFP}(\mathrm{IU} / \mathrm{ml})$ & $45[1-2479]$ \\
\hline \multicolumn{2}{|c|}{$\begin{array}{l}\text { Abbreviation: BCLC, Barcelona Clinic Liver Cancer; HBV, HCV, hepatitis B and } \\
\text { hepatitis C virus; HCC, hepatocellular carcinoma; AFP, alpha fetoprotein; WBC, } \\
\text { white blood cells; RBC, red blood cells; HCT, hematocrit; PLT, platelets; AST and } \\
\text { ALT, aspartate and alanine amino transferase; IU, international unit; NA, not } \\
\text { applicable. }\end{array}$} \\
\hline
\end{tabular}

\section{Quantification of relative gene expression by quantitative real-time PCR}

Total RNA was extracted from the 38 tumour and adjacent non-tumour tissue pairs by Trizol reagent (Life Technologies, Carlsbad, CA, USA) according to the protocol recommended by the manufacturer. Extracted total RNA samples were reverse transcribed into cDNA by using the QuantiTect Reverse Transcription Kit (Qiagen, Hilden, Germany). cDNA was quantified by real-time PCR with GAPDH (glyceraldehyde-3-phosphate dehydrogenase) used as reference gene. Sequences of primers used in this study are presented in Table 2. Quantitative realtime PCR reactions were carried out in a volume of $25 \mu \mathrm{l}$ containing $12.5 \mu \mathrm{l} 2 \times$ SYBR Green PCR master mix (Bioline, Luckenwalde, Germany), $0.5 \mu \mathrm{M}$ primer pairs specific for the target genes and the reference gene, $5 \mathrm{ng}$ cDNA samples and RNase-free water up to the $25 \mu$ reaction volume. Thermal cycling conditions were $95{ }^{\circ} \mathrm{C}$ for 2 minutes for initial activation, followed by 45 cycles of denaturation at $95{ }^{\circ} \mathrm{C}$ for $5 \mathrm{sec}$ and annealing and extension at $58{ }^{\circ} \mathrm{C}$ for $20 \mathrm{sec}$. Melting curve analyses starting from $58^{\circ} \mathrm{C}$ to $85^{\circ} \mathrm{C}$ were performed after each run to confirm specificity of the PCR products. All reactions were performed in duplicates and repeated twice using the LightCycler ${ }^{\circledR} 480$ real-time PCR system (Roche, Basel, Switzerland). Relative expression of target genes was normalized to the expression of GAPDH based on the $\triangle \mathrm{Ct}$ method.

Table 2: Primers used for this study

\begin{tabular}{|c|c|c|c|}
\hline Primer & Sequence & Target gene & Reference \\
\hline USP18_F & $\begin{array}{l}\text { 5'- TGT CAG TCC ATC CTG GCT GAG TC } \\
-3^{\prime}\end{array}$ & USP18 & This study \\
\hline USP18_R & $\begin{array}{l}\text { 5'- CAC CTG AAT CAA GGA GTT AAG } \\
\text { GCA GC - } 3^{\prime}\end{array}$ & & \\
\hline UBA1_F & 5'- GCT CGC CGC TGT CCA AGA AAC -3' & UBA1 & This study \\
\hline$U B A 1 \_R$ & $\begin{array}{l}\text { 5'- GGG AGT AAA GGC CCT CGT CTA } \\
\text { TGT C - } 3^{\prime}\end{array}$ & & \\
\hline$E F P \_F$ & 5'- CGT GGA GTG GTT CAA CAC -3' & EFP & [25] \\
\hline$E F P \_R$ & 5'- GAG CAG ATG GAG AGT GTG G -3' & (TRIM25) & \\
\hline HERC5_F & 5'- AAC CTG CAT GGG CAG CTT GG -3' & HERC5 & [36] \\
\hline HERC5_R & $5^{\prime}$ - TGT GGG CTT CTC CGG CAG AA - $3^{\prime}$ & & \\
\hline$U B C \_F$ & 5'-GGG TCG CAG TTC TTG TTT GT-3' & $U B C$ & [35] \\
\hline$U B C \_R$ & 5'-TCC AGC AAA GAT CAG CCT CT-3' & & \\
\hline GAPDH_F & $5^{\prime}$ - TGA ACG GGA AGC TCA CTG G -3' & GADPH & [25] \\
\hline GAPDH_R & 5'- TCC ACC ACC CTG TTG CTG TA-3' & & \\
\hline
\end{tabular}

\section{Statistical analysis}

Quantitative parameters including laboratory tests and relative gene expression values are given as means with standard deviation or medians with ranges where appropriate. The relative gene expression between tumour and adjacent non-tumour tissues was compared using the Wilcoxon signed ranks test. Spearman's rank correlation coefficient was used to analyze the correlation of relative gene expression between two genes or between expression of the genes with clinical parameters. The SPSS software version 22.0 (SPSS Statistics, IBM, Armonk, NY, the USA) was used for all statistical analyses; the significance level was set at $P<0.05$.

\section{Results}

\section{Gene expression in HCC patients}

Relative expression of the five genes EFP, HERC5, UBA1, UBC and USP18, which are related to ISGylation, was quantified and compared between HCC tumour and adjacent non-tumour tissues. Expression of EFP, HERC5, UBA1, and USP18 was significantly higher in HCC tumour tissues compared to the adjacent non-tumour tissues $(P=0.006,0.012$, 0.02 and 0.039 , respectively). No significant difference of $U B C$ expression was observed between HCC tumour tissues and non-tumour tissues $(P=0.91)$ (Figure 1). These results indicate that EFP, HERC5, UBA1, and USP18 are upregulated in HCC tumours and might be associated with the development of HCC. 
We then examined whether gene expression was associated with progression of HCC by comparing their expression between different BCLC stages. However, expression of the genes did not differ between stage A and B HCC tissues as well as between the corresponding non-tumour tissues $(P>0.05)$. BCLC stages $C$ and $D$ did not occur among the study participants.

\section{Correlation of mRNA expression between genes}

We analysed the correlations of mRNA expression in both HCC tumour and non-tumour tissues. In HCC tissues, gene expression was strongly correlated with each other (Spearman's rho >0.85; $P<0.0001)$. The strongest correlation was observed between expression of EFP and HERC5 (Spearman's rho $=0.98 ; P<0.0001)$, followed by expression of HERC5 and USP18 (Spearman's rho $=0.95 ; P<0.0001$ ) and of EFP and UBA1 (Spearman's rho $=0.94$; $P<0.0001$ ) (Table 3). In non-tumour tissues, EFP expression was strongly correlated with that of HERC5, UBA1 and USP18 (Spearman's rho $=0.81,0.89$ and $0.75 ; P<0.0001$, respectively). A similar result was observed when the correlation of HERC5 expression with that of UBA1 and USP18 was analysed (Spearman's rho=0.76; $P<0.0001$ ) (Table 3). The correlation of $U B C$ mRNA expression with EFP, UBA1 and USP18 was moderately positive (Spearman's rho $=0.58,49$ and 54; $P<0.0001,0.003$ and 0.0007, respectively). A similar correlation was seen between
UBA1 and USP18 (Spearman's rho $=0.56 ; P=0.0005$ ). These results indicate that the expression pattern of the genes under study differed between HCC tumour and adjacent non-tumour tissues.

Table 3: Correlation of relative expression between genes in tumour and adjacent non-tumour tissues

\begin{tabular}{|c|c|c|c|c|c|c|c|c|c|c|}
\hline Gene & $E F P$ & & HER & & $U B A$ & & $U B C$ & & USP & \\
\hline & $r_{s}$ & $P$ & $r_{s}$ & $P$ & $r_{s}$ & $P$ & $r_{s}$ & $P$ & $r_{s}$ & $P$ \\
\hline$E F P$ & & & 0.98 & $<0.0001$ & 0.94 & $<0.0001$ & 0.91 & $<0.0001$ & 0.93 & $<0.0001$ \\
\hline HERC5 & 0.81 & $<0.0001$ & & & 0.91 & $<0.0001$ & 0.91 & $<0.0001$ & 0.95 & $<0.0001$ \\
\hline$U B A 1$ & 0.89 & $<0.0001$ & 0.76 & $<0.0001$ & & & 0.9 & $<0.0001$ & 0.89 & $<0.0001$ \\
\hline$U B C$ & 0.58 & $<0.0001$ & 0.69 & $<0.0001$ & 0.49 & 0.003 & & & 0.85 & $<0.0001$ \\
\hline USP18 & 0.75 & $<0.0001$ & 0.76 & $<0.0001$ & 0.56 & 0.0005 & 0.54 & 0.0007 & & \\
\hline
\end{tabular}

Spearman's rho $\left(\mathrm{r}_{\mathrm{s}}\right)$ calculated by Spearman's rank correlation coefficient. $\mathrm{r}_{\mathrm{s}}$ and $P$ values in the lower-left area are the correlation of relative expression between genes in adjacent non-tumour tissues. $r_{\mathrm{s}}$ and $P$ values in the upper-right area are the correlation of relative expression between genes in HCC tumour tissues.

\section{Correlation of gene expression with laboratory parameters}

We analysed the correlations of relative gene expression with laboratory parameters of HCC patients. Expression of EFP, HERC5 and UBA1 in non-tumour tissues were positively correlated with direct bilirubin levels (Spearman's rho $=0.31,0.33$ and $0.45 ; P=0.06,0.05$ and 0.01, respectively) (Figure 2, upper panel). Expression of $U B C$ in non-tumour tissues was negatively correlated with AST and total bilirubin levels (Spearman's rho $=-0.39$ and -0.31 ; $P=0.017$ and 0.065 , respectively), but positively correlated with albumin levels (Spearman's rho $=0.36$; $P=0.03$ ) (Figure 2, middle panel). In addition,
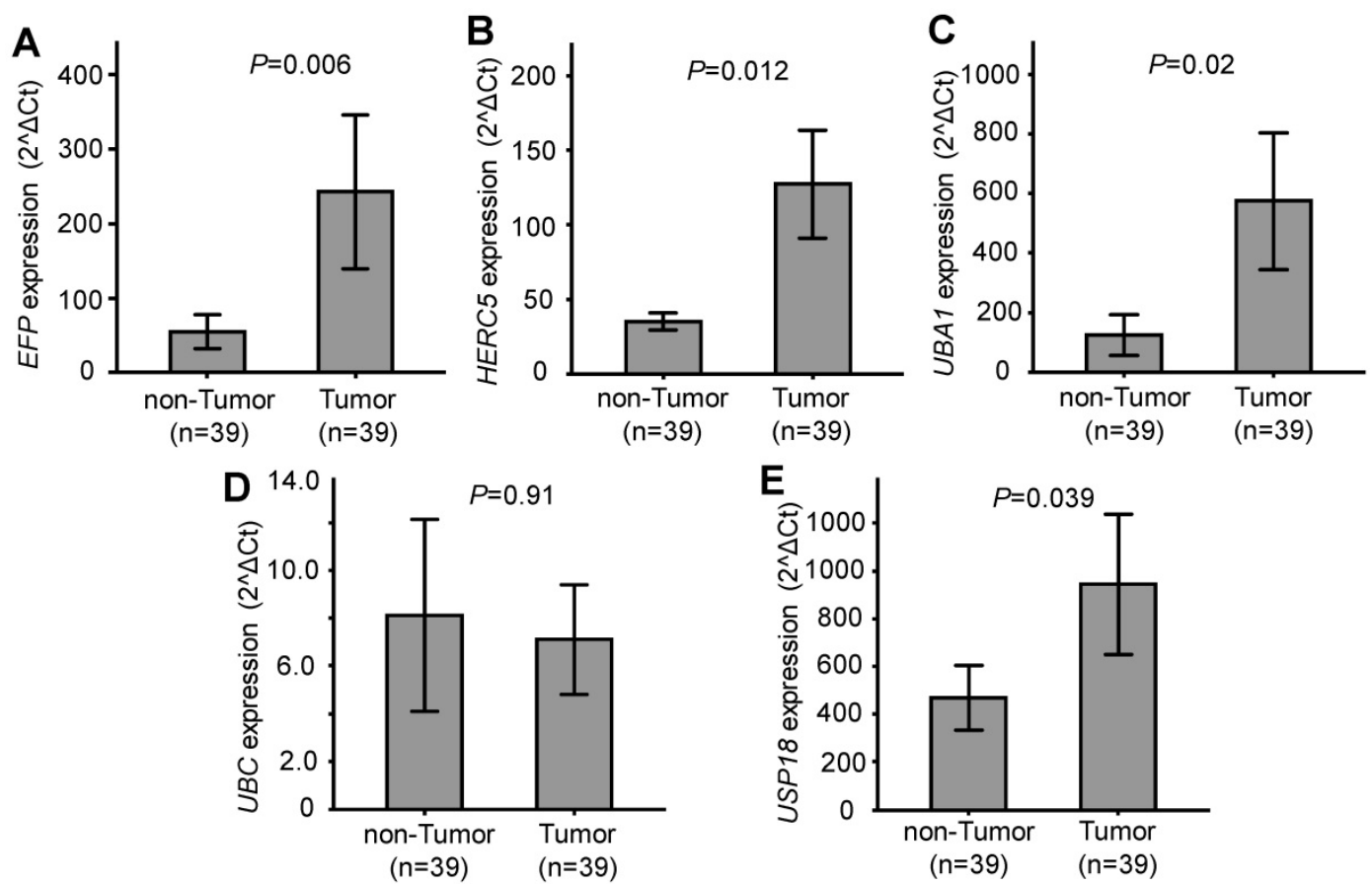

Figure 1: Gene expression in tumour and adjacent non-tumour tissues. A, B, C, D and E: Relative expression of EFP, HERC5, UBA I, UBC and USPI8, respectively. $P$ values were calculated by Wilcoxon signed ranks test. 

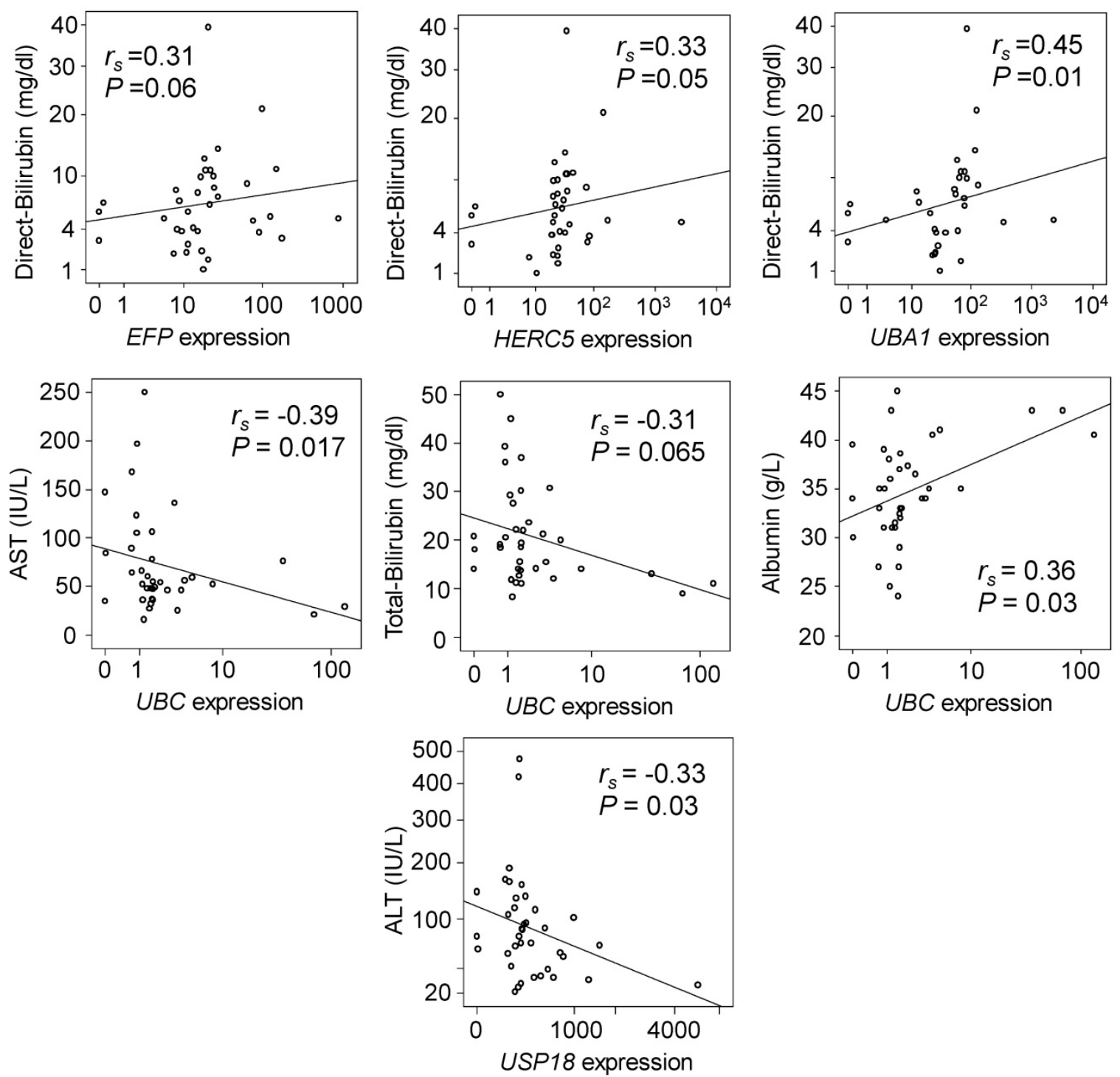

Figure 2: Correlation of gene expression with clinical parameters. Spearman's rho $\left(r_{s}\right)$ and $P$ values presented in the figures were calculated by Spearman's rank correlation coefficient.

expression of USP18 in non-tumour tissues was negatively correlated with ALT levels (Spearman's rho $=-0.33, P=0.03$ ) (Figure 2, lower panel). However, in HCC tumour tissues no significant correlation was observed.

\section{Discussion}

ISGylation plays a key role both in immunity against infections and in the formation and progression of tumours by affecting a wide range of target proteins [10]. A number of enzymes involved in the ISGylation process, namely E3 ligases (HERC5 and EFP/TRIM25) and USP18 have been shown to be associated with cancers via ISGylation-dependent and -independent mechanisms [15-18]. We have previously shown that ISG15 expression is associated with HBV-related liver diseases, including HCC [23]. Here we show that expression of E3 ligases (HERC5 and EFP/TRIM25), UBA1 and USP18 is significantly upregulated in HCC tumours compared to adjacent non-tumour liver tissues. In addition, the expression levels of the genes in non-tumour tissues is associated with several clinical parameters. Our results indicate that E3 ligases, UBA1 and USP18 are associated with HCC development and may possibly be considered as targets in the treatment of HCC.

The upregulation of EFP, HERC5, UBA1 and USP18 in HCC tissues and the different patterns of correlation of the genes in HCC tumour and in adjacent non-tumour tissues suggest that these genes may play a role in the pathogenesis of HCC. Studies have shown that EFP is closely associated with breast cancer [6, 24]. EFP regulates metastasis of breast cancer through its E3 ubiquitin ligase activity rather than through estrogen signalling [24]. Expression of EFP is upregulated in breast and gastric cancers but 
downregulated in endometrial cancer $[15,25,26]$. High EFP expression is associated with a poor prognosis of breast cancer [25], associated with advanced disease in human epithelial ovarian cancer [16], and high EFP expression is a prognostic factor in prostate cancer [26]. It has also been shown that EFP increases proliferation and survival of prostate cancer cells by affecting p53 signals [26]. EFP is also associated with HCC progression through degrading the metastasis-associated 1 protein (MTA-1) [27, 28]. Here we show that EFP is significantly upregulated in HCC tissue compared to non-tumour liver tissues and EFP expression in non-tumour tissues is correlated with the levels of direct bilirubin.

HERC5 is the dominant E3 enzyme for ISGylation and has been shown to be highly expressed in HCC tissues and in vitro [18]. It contributes to immune evasion in HCC and may be a prognostic biomarker for recurrent HCC after liver transplantation [17]. In line with a previous study [18], we also observed upregulation of HERC5 in HCC tumour tissues and a correlation of HERC5 expression with direct bilirubin levels in non-tumour tissues. Although UBA1 has been considered as a potential target gene for cancer therapy, especially for acute myeloid leukemia [29], the role of this gene in the pathogenesis of HCC had not been elucidated so far. To the best of our knowledge, this study for the first-time analyses UBA1 expression in HCC tumour and adjacent non-tumour tissues and points towards its correlation with direct bilirubin levels.

In agreement with a previous report [21], our study also shows that USP18 is significantly upregulated in HCC tissues compared to corresponding non-tumour tissues. USP18 is an important factor in the immune response directed against HBV replication and its overexpression promotes growth of HCC cells [21]. Our results underline the crucial role of USP18 in HCC tumourigenesis; however, the exact mechanism by which USP18 is involved in HCC development remains unclear, as USP18 exhibits diverse functions. It influences cell cycle progression by interacting with S-phase kinase-associated protein 2 (SKP2) [30] and plays an important role in inhibition of $\mathrm{HBV}$ replication through activation of the JAK/STAT signalling pathway [31], independent of ISG15 and ISGylation [32]. Recently, expression of USP18 mRNA in peripheral blood mononuclear cells (PBMCs) has been proposed as a predictive factor for monitoring treatment of HBV patients with interferon-alpha [33]. Another mechanism by which USP18 might contribute to tumour formation and progression in lung cancer is that USP18 can influence functionality of the cyclin D1 and KRAS proteins [20, 22]. Although
USP18 has been shown to inhibit tumourigenesis [34], further studies are required to describe in more detail the precise role of USP18 in HBV-related HCC.

$U B C$ expression is upregulated in lung cancer [35]. Although the relative expression of $U B C$ does not differ significantly between HCC tumour and non-tumour tissues, $U B C$ expression in non-tumour tissues is correlated with AST levels, total bilirubin and albumin, indicating that UBC may not significantly contribute to the pathogenesis of HCC, but rather plays a role in liver functions. Limitations of this study apply to the rather small number of HCC patients, particularly the small number of HCC patients in BCLC stages $\mathrm{C}$ and $\mathrm{D}$, implying the impossibility to perform association analyses of gene expression with HCC progression.

In conclusion, our study shows that expression of EFP, HERC5, UBA1 and USP18 genes, upregulated in tumour tissues of HCC patients, is correlated with liver function parameters and, thus, may be associated with the pathogenesis of liver cancer.

\section{Acknowledgements}

We would like to thank all patients for their participation. This study was funded by the Vietnam National Foundation for Science and Technology Development (NAFOSTED; grant number 108.02-2017.15) to Hoang Van Tong. The funder has no role in the study design, data collection and analysis, decision to publish or preparation of the manuscript.

\section{Competing Interests}

The authors have declared that no competing interest exists.

\section{References}

1. El-Serag HB. Hepatocellular carcinoma. N Engl J Med. 2011; 365: 1118-27.

2. Bray F, Ferlay J, Soerjomataram I, Siegel RL, Torre LA, Jemal A. Global cancer statistics 2018: GLOBOCAN estimates of incidence and mortality worldwide for 36 cancers in 185 countries. CA Cancer J Clin. 2018; 68: 394-424.

3. Villanueva A. Hepatocellular Carcinoma. N Engl J Med. 2019; 380: 1450-62.

4. Bishayee A. The role of inflammation and liver cancer. Adv Exp Med Biol. 2014; 816: 401-35.

5. Rijckborst V, Janssen HL. The Role of Interferon in Hepatitis B Therapy. Curr Hepat Rep. 2010; 9: 231-8.

6. Jeon YJ, Yoo HM, Chung CH. ISG15 and immune diseases. Biochim Biophys Acta. 2010; 1802: 485-96.

7. Lenschow DJ. Antiviral Properties of ISG15. Viruses 2010; 2: 2154-68

8. Perng YC, Lenschow DJ. ISG15 in antiviral immunity and beyond. Nat Rev Microbiol. 2018; 16: 423-39.

9. Campbell JA, Lenschow DJ. Emerging roles for immunomodulatory functions of free ISG15. Journal of interferon \& cytokine research: the official journal of the International Society for Interferon and Cytokine Research. 2013; 33: 728-38.

10. Villarroya-Beltri C, Guerra S, Sanchez-Madrid F. ISGylation - a key to lock the cell gates for preventing the spread of threats. J Cell Sci. 2017; 130: 2961-9.

11. Huang YF, Wee S, Gunaratne J, Lane DP, Bulavin DV. Isg15 controls p53 stability and functions. Cell Cycle. 2014; 13: 2200-10.

12. Tecalco-Cruz AC, Cortes-Gonzalez CC, Cruz-Ramos E, Ramirez Jarquin JO, Romero-Mandujano AK, Sosa-Garrocho M. Interplay between interferon-stimulated gene 15/ISGylation and interferon gamma signaling in breast cancer cells. Cell Signal. 2019; 54: 91-101. 
13. Yeung TL, Tsai CC, Leung CS, Au Yeung CL, Thompson MS, Lu KH, et al. ISG15 Promotes ERK1 ISGylation, CD8+ T Cell Activation and Suppresses Ovarian Cancer Progression. Cancers (Basel). 2018; 10.

14. Yoo L, Yoon AR, Yun CO, Chung KC. Covalent ISG15 conjugation to CHIP promotes its ubiquitin E3 ligase activity and inhibits lung cancer cell growth in response to type I interferon. Cell Death Dis. 2018; 9: 97.

15. Dai H, Zhao S, Xu L, Chen A, Dai S. Expression of Efp, VEGF and bFGF in normal, hyperplastic and malignant endometrial tissue. Oncol Rep. 2010; 23 : 795-9.

16. Sakuma M, Akahira J, Suzuki T, Inoue S, Ito K, Moriya T, et al. Expression of estrogen-responsive finger protein (Efp) is associated with advanced disease in human epithelial ovarian cancer. Gynecol Oncol. 2005; 99: 664-70.

17. Xue F, Higgs BW, Huang J, Morehouse C, Zhu W, Yao X, et al. HERC5 is a prognostic biomarker for post-liver transplant recurrent human hepatocellular carcinoma. J Transl Med. 2015; 13: 379.

18. Wang Y, Ding Q, Xu T, Li CY, Zhou DD, Zhang L. HZ-6d targeted HERC5 to regulate p53 ISGylation in human hepatocellular carcinoma. Toxicol Appl Pharmacol. 2017; 334: 180-91.

19. Tan Y, Zhou G, Wang X, Chen W, Gao H. USP18 promotes breast cancer growth by upregulating EGFR and activating the AKT/Skp2 pathway. Int J Oncol. 2018; 53: 371-83.

20. Mustachio LM, Lu Y, Tafe LJ, Memoli V, Rodriguez-Canales J, Mino B, et al. Deubiquitinase USP18 Loss Mislocalizes and Destabilizes KRAS in Lung Cancer. Mol Cancer Res. 2017; 15: 905-14.

21. Cai J, Liu T, Jiang X, Guo C, Liu A, Xiao X. Downregulation of USP18 inhibits growth and induces apoptosis in hepatitis B virus-related hepatocellular carcinoma cells by suppressing BCL2L1. Exp Cell Res. 2017; 358: 315-22.

22. Guo Y, Chinyengetere F, Dolinko AV, Lopez-Aguiar A, Lu Y, Galimberti F, et al. Evidence for the ubiquitin protease UBP43 as an antineoplastic target. Mol Cancer Ther. 2012; 11: 1968-77.

23. Hoan NX, Van Tong H, Giang DP, Toan NL, Meyer CG, Bock CT, et al. Interferon-stimulated gene 15 in hepatitis B-related liver diseases. Oncotarget 2016; 7: 67777-87.

24. Walsh LA, Alvarez MJ, Sabio EY, Reyngold M, Makarov V, Mukherjee S, et al. An Integrated Systems Biology Approach Identifies TRIM25 as a Key Determinant of Breast Cancer Metastasis. Cell Rep. 2017; 20: 1623-40.

25. Suzuki T, Urano T, Tsukui T, Horie-Inoue K, Moriya T, Ishida T, et al. Estrogen-responsive finger protein as a new potential biomarker for breast cancer. Clin Cancer Res. 2005; 11: 6148-54.

26. Zhu Z, Wang Y, Zhang C, Yu S, Zhu Q, Hou K, et al. TRIM25 blockade by RNA interference inhibited migration and invasion of gastric cancer cells through TGF-beta signaling. Sci Rep. 2016; 6: 19070.

27. Zang HL, Ren SN, Cao H, Tian XF. The ubiquitin ligase TRIM25 inhibits hepatocellular carcinoma progression by targeting metastasis associated 1 protein. IUBMB Life 2017; 69: 795-801.

28. Li YH, Zhong M, Zang HL, Tian XF. The E3 ligase for metastasis associated 1 protein, TRIM25, is targeted by microRNA-873 in hepatocellular carcinoma. Exp Cell Res. 2018; 368: 37-41.

29. Barghout $\mathrm{SH}$, Patel PS, Wang $\mathrm{X}, \mathrm{Xu}$ GW, Kavanagh $\mathrm{S}$, Halgas $\mathrm{O}$, et al. Preclinical evaluation of the selective small-molecule UBA1 inhibitor, TAK-243, in acute myeloid leukemia. Leukemia 2019; 33: 37-51.

30. Vuillier F, Li Z, Commere PH, Dynesen LT, Pellegrini S. USP18 and ISG15 coordinately impact on SKP2 and cell cycle progression. Sci Rep. 2019; 9: 4066.

31. Li L, Lei QS, Zhang SJ, Kong LN, Qin B. Suppression of USP18 Potentiates the Anti-HBV Activity of Interferon Alpha in HepG2.2.15 Cells via JAK/STAT Signaling. PLoS One 2016; 11: e0156496.

32. Kim JH, Luo JK, Zhang DE. The level of hepatitis B virus replication is not affected by protein ISG15 modification but is reduced by inhibition of UBP43 (USP18) expression. Journal of immunology (Baltimore, Md: 1950). 2008; 181: 6467-72.

33. Liu W, Liang H, Wang S, Wu C, Liu Y, Liu Y, et al. Transcriptional response of USP18 predicts treatment outcomes of interferon-alpha in HBeAg-positive chronic hepatitis B patientsefere. J Viral Hepat. 2019.

34. Hong B, Li H, Lu Y, Zhang M, Zheng Y, Qian J, et al. USP18 is crucial for IFN-gamma-mediated inhibition of B16 melanoma tumorigenesis and antitumor immunity. Mol Cancer. 2014; 13: 132.

35. Tang Y, Geng Y, Luo J, Shen W, Zhu W, Meng C, et al. Downregulation of ubiquitin inhibits the proliferation and radioresistance of non-small cell lung cancer cells in vitro and in vivo. Sci Rep. 2015; 5: 9476.

36. Ashley RL, Henkes LE, Bouma GJ, Pru JK, Hansen TR. Deletion of the Isg15 gene results in up-regulation of decidual cell survival genes and down-regulation of adhesion genes: implication for regulation by IL-1beta. Endocrinology 2010; 151: 4527-36. 